\title{
Dense gas in low-metallicity galaxies
}

\author{
J. Braine ${ }^{1}$, Y. Shimajiri ${ }^{2}$, P. André2 ${ }^{2}$ S. Bontemps ${ }^{1}, \mathrm{Yu} \mathrm{Gao}^{3,4}$, Hao Chen ${ }^{5,6,7}$, and C. $\mathrm{Kramer}^{8}$ \\ ${ }^{1}$ Laboratoire d'Astrophysique de Bordeaux, Univ. Bordeaux, CNRS, B18N, Allée Geoffroy Saint-Hilaire, 33615 Pessac, France \\ e-mail: jonathan. braine@u-bordeaux. fr \\ ${ }^{2}$ Laboratoire AIM, CEA/DRF - CNRS - Université Paris Diderot, IRFU/Service d'Astrophysique, C.E. Saclay, Orme des Merisiers, \\ 91191 Gif-sur-Yvette, France \\ 3 Purple Mountain Observatory, Chinese Academy of Sciences, 2 West Beijing Road, 210008 Nanjing, PR China \\ ${ }^{4}$ Key Laboratory of Radio Astronomy, Chinese Academy of Sciences, 210008 Nanjing, PR China \\ 5 School of Astronomy and Space Science, Nanjing University, 210093 Nanjing, PR China \\ 6 Key Laboratory of Modern Astronomy and Astrophysics, Nanjing University, 210093 Nanjing, PR China \\ 7 Collaborative Innovation Center of Modern Astronomy and Space Exploration, 210093 Nanjing, PR China \\ 8 Instituto Radioastronoma Milimétrica, Av. Divina Pastora 7, Nucleo Central, 18012 Granada, Spain
}

Received 23 September 2016 / Accepted 27 October 2016

\begin{abstract}
Stars form out of the densest parts of molecular clouds. Far-IR emission can be used to estimate the star formation rate (SFR) and high dipole moment molecules, typically HCN, trace the dense gas. A strong correlation exists between HCN and far-IR emission, with the ratio being nearly constant, over a large range of physical scales. A few recent observations have found HCN to be weak with respect to the far-IR and CO in subsolar metallicity (low-Z) objects. We present observations of the Local Group galaxies M 33, IC 10, and NGC 6822 with the IRAM $30 \mathrm{~m}$ and NRO $45 \mathrm{~m}$ telescopes, greatly improving the sample of low- $Z$ galaxies observed. $\mathrm{HCN}, \mathrm{HCO}^{+}$, $\mathrm{CS}, \mathrm{C}_{2} \mathrm{H}$, and $\mathrm{HNC}$ have been detected. Compared to solar metallicity galaxies, the nitrogen-bearing species are weak (HCN, $\mathrm{HNC}$ ) or not detected $\left(\mathrm{CN}, \mathrm{HNCO}, \mathrm{N}_{2} \mathrm{H}^{+}\right)$relative to far-IR or $\mathrm{CO}$ emission. $\mathrm{HCO}^{+}$and $\mathrm{C}_{2} \mathrm{H}$ emission is normal with respect to $\mathrm{CO}$ and far-IR. While ${ }^{13} \mathrm{CO}$ is the usual factor 10 weaker than ${ }^{12} \mathrm{CO}, \mathrm{C}^{18} \mathrm{O}$ emission was not detected down to very low levels. Including earlier data, we find that the $\mathrm{HCN} / \mathrm{HCO}^{+}$ratio varies with metallicity $(\mathrm{O} / \mathrm{H})$ and attribute this to the sharply decreasing nitrogen abundance. The dense gas fraction, traced by the $\mathrm{HCN} / \mathrm{CO}$ and $\mathrm{HCO}^{+} / \mathrm{CO}$ ratios, follows the SFR but in the low-Z objects the $\mathrm{HCO}^{+}$is much easier to measure. Combined with larger and smaller scale measurements, the $\mathrm{HCO}^{+}$line appears to be an excellent tracer of dense gas and varies linearly with the SFR for both low and high metallicities.
\end{abstract}

Key words. galaxies: individual: M 33 - galaxies: individual: IC 10 - galaxies: individual: NGC 6822 - Local Group galaxies: ISM - stars: formation

\section{Introduction}

Stars are believed to condense out of prestellar cores within the densest parts, clumps and/or filaments above $A_{v} \sim 8$ or $n_{\mathrm{H} 2} \sim 1-2 \times 10^{4} \mathrm{~cm}^{-3}$ (André et al. 2010; Lada et al. 2010) of giant molecular clouds (GMCs). To study the link between gas and star formation, it is important to observe molecules which emit preferentially in these environments. $\mathrm{HCN}$ and $\mathrm{HCO}^{+}$are used to trace dense molecular gas because their dipole moments are high, such that they are thermalized by collisions only at high densities $\left(n\left(\mathrm{H}_{2}\right)>10^{4} \mathrm{~cm}^{-3}\right)$ even for the lowest transitions. If star formation proceeds in a quasi-universal manner in all dense clumps/filaments (André et al. 2014), then we expect the star formation rate (SFR) to be strongly and linearly correlated with the dense gas mass. In terms of observables, we expect the farinfrared (FIR) emission to follow that of $\mathrm{HCN}$ and $\mathrm{HCO}^{+}$. A large number of articles (e.g. Gao \& Solomon 2004a,b; Wu et al. 2005; Gao et al. 2007; Baan et al. 2008; Graciá-Carpio et al. 2008; Wu et al. 2010; García-Burillo et al. 2012; Liu \& Gao 2010; Lada et al. 2012; Kepley et al. 2014) have studied the link between $\mathrm{HCN}$, and $\mathrm{HCO}^{+}$in more recent work, with FIR luminosities in a variety of environments. They tend to show that FIR and $\mathrm{HCN}$ emission (and often $\mathrm{HCO}^{+}$) are closely linked. Recent work by Chen et al. (2015) and Usero et al. (2015) showed that $\mathrm{HCN}$ emission, both with respect to the total molecular gas mass (as traced by CO) and to the SFR (as traced by FIR emission), was significantly stronger in galactic nuclei as compared to galactic disks.

Both $\mathrm{HCN}$ and $\mathrm{HCO}^{+}$have low abundances, orders of magnitude lower than $\mathrm{CO}$, so their abundances can be significantly modified by changes in the chemical network leading to their formation (e.g. López-Sepulcre et al. 2010), and these changes are not necessarily be linked to star formation. Unlike $\mathrm{HCN}, \mathrm{HCO}^{+}$ is a molecular ion and affected by the ionization equilibrium. In addition, the collisional cross-section of $\mathrm{HCO}^{+}$is much larger than that of HCN. Both molecules are linear and thus are described by a single rotational quantum number. Elemental abundance ratios are not always constant and can affect the production of molecules.

In particular, the nitrogen abundance tends to decrease more quickly than the oxygen or carbon in low-metallicity galaxies. In galactic disks, the $\mathrm{HCN} / \mathrm{CO}$ and $\mathrm{HCO}^{+} / \mathrm{CO}$ line intensity ratios (1-0 transition, expressed in $\mathrm{K} \mathrm{km} \mathrm{s}^{-1}$ ) are about $2 \%$, although in galactic nuclei and starburst galaxies these ratios tend to be higher (see references in first paragraph and Brouillet et al. 2005 for M 31 and Kuno et al. 1995, for M 51). Recently, Buchbender et al. (2013) showed that in the roughly half-solar metallicity galaxy M 33, the emission from the dense gas tracers $\mathrm{HCN}$ and $\mathrm{HCO}^{+}$was weak relative to $\mathrm{CO}$, particularly 
HCN. Gratier et al. (2010a) actually failed to detect HCN in the CO-bright region Hubble $\mathrm{V}$ in the Local Group low-metallicity galaxy NGC 6822, with a $1 \sigma$ limit 180 times weaker than the $\mathrm{CO}$. The variations between $\mathrm{HCO}^{+}$and $\mathrm{HCN}$ show that new observing programs require both tracers as $\mathrm{HCO}^{+}$is probably less affected by metallicity variations and tends to be stronger than $\mathrm{HCN}$ in disks but not in nuclei.

Descending the mass/morphology/metallicity sequence from M 31 to M 33 to the Magellanic Clouds (MC), the $\mathrm{HCO}^{+} / \mathrm{HCN}$ ratio increases from 1.2 in $\mathrm{M} 31$ (Brouillet et al. 2005, particularly Fig. 3), 1.5 (range from 1.1 to 2.5) in $\mathrm{M} 33$ (Buchbender et al. 2013), to $\sim 1.8$ in the Large MC (Chin et al. 1997) and 3 in the Small MC (Chin et al. 1998, single position). Within $M$ 31, a rather rough trend can be seen with radius, with the $\mathrm{HCO}^{+} / \mathrm{HCN}$ ratio increasing from 1 in the inner parts to $\sim 1.4$ in the outer disk. Are these variations in the $\mathrm{HCN} / \mathrm{CO}$ ratio and the $\mathrm{HCO}^{+} / \mathrm{HCN}$ ratio due to metallicity?

Recent comparisons between Galactic and extragalactic studies of the SF rate (Heiderman et al. 2010; Lada et al. 2012) suggest that there may a quasi-universal "star formation law" such that dense gas $\left(\geq 10^{4} \mathrm{~cm}^{-3}\right)$ is converted into stars at a constant rate with a constant stellar initial mass function (IMF). It has been proposed that this may be the result of the quasiuniversal filamentary structure of molecular clouds (André et al. 2014).

In this work, our goal is to understand how to use dense gas tracers in low-metallicity galaxies. More precisely, we wish to measure the variation with metallicity of the link between the dense gas tracers $\mathrm{HCN}$ and $\mathrm{HCO}^{+}$and the FIR and $\mathrm{CO}$ emission. In order to keep observing times reasonable, only positions/objects with $\mathrm{CO}$ lines brighter than $\sim 0.5 \mathrm{~K}\left(T_{\mathrm{mb}}\right.$ at $\sim 20^{\prime \prime}$ resolution) were observed. In order that the linear resolution be comparable to the size of a molecular cloud or cloud complex $(\sim 100 \mathrm{pc})$, we restricted our observations to Local Group galaxies which cover a rather complete range in metallicity. Most extragalactic studies focus on the central regions - whether by choice or simply due to the single pointing per object - where it is known that gas is on average denser. By observing Local Group objects, we observe identifiable positions in disks where a wealth of other data is available, such as HI column densities and several tracers of the SFR (H $\alpha$, FIR, FUV...). Detailed analyses of the biases and validity of a broad variety of SFR tracers can be found in Boquien et al. (2011) and Galametz et al. (2013).

While the major starbursts (ULIRGs) observed in Gao \& Solomon (e.g. 2004b,a) likely have a solar metallicity, this is not the general case for distant galaxies. Only by studying the role of metallicity in local galaxies can we evaluate the "universality" of the "dense gas - star formation" relation and its tentative application to high-redshift galaxies.

In addition to $\mathrm{HCN}(1-0)$ and $\mathrm{HCO}^{+}(1-0)$, the $\mathrm{HNC}(1-0)$, $\mathrm{N}_{2} \mathrm{H}^{+}(1-0), \mathrm{CS}(2-1)$, and $\mathrm{CCH}$ lines were observed and, with the exception of $\mathrm{N}_{2} \mathrm{H}^{+}$, detected. The $\mathrm{HCN} / \mathrm{HNC}$ ratio is typically about 3 in the Magellanic Clouds (Chin et al. 1997) but closer to unity in e.g. IC 342 (Meier \& Turner 2005). Galactic observations suggest that the $\mathrm{HCN} / \mathrm{HNC}$ ratio can be used as a probe of physical conditions (HCN and HNC dipole moments are virtually identical). The $\mathrm{HCN} / \mathrm{HNC}$ ratio is typically greater than unity, sometimes much greater, in warm GMC cores (e.g. Hirota et al. 1998) but can be as low as 0.1 or 0.2 in dark cloud cores (e.g. Tennekes et al. 2006, and references therein). Thus, detected variations in this ratio are likely a sensitive temperature indicator. The $\mathrm{N}_{2} \mathrm{H}^{+}$line is a classical tracer of cool and dense regions in the Galaxy as it depletes only very weakly onto dust grains. With 2 nitrogen atoms, $\mathrm{N}_{2} \mathrm{H}^{+}$should be strongly affected
Table 1. Sources and positions observed in M 33, IC 10, and NGC 6822.

\begin{tabular}{lllll}
\hline \hline Source & $\begin{array}{l}\text { Distance } \\
(\mathrm{kpc})\end{array}$ & $\begin{array}{l}\text { Mtallicity } \\
\left(Z_{\text {sol }}\right)\end{array}$ & $\begin{array}{l}\text { RA } \\
(\mathrm{J} 2000)\end{array}$ & $\begin{array}{l}\text { Dec } \\
(\mathrm{J} 2000)\end{array}$ \\
\hline M 33a & 840 & 0.5 & $01: 34: 34.5$ & $30: 46: 30$ \\
M 33b & 840 & 0.5 & $01: 34: 33.56$ & $30: 46: 46$ \\
M 33c & 840 & 0.5 & $01: 34: 32.3$ & $30: 46: 58$ \\
IC 10b8 & 950 & 0.35 & $0: 20: 21.6$ & $59: 17: 09$ \\
IC 10b9 & 950 & 0.35 & $0: 20: 22.9$ & $59: 21: 18$ \\
IC 10b11 & 950 & 0.35 & $0: 20: 27.7$ & $59: 17$ \\
N6822HubV & 490 & 0.3 & $19: 44: 52.80$ & $-14: 43: 11$ \\
\hline
\end{tabular}

Notes. Distances are from Galleti et al. (2004) for M 33, Hunter (2001) for IC 10, Mateo (1998) for NGC 6822. Metallicities are from Magrini et al. (2009) for M 33, Magrini \& Gonçalves (2009) for IC 10, Skillman et al. (1989) for NGC 6822. Positions are chosen from our data (Gratier et al. 2010b; Druard et al. 2014; Gratier et al. 2010a) for M 33 and NGC 6822 and Leroy et al. (2006) for IC 10.

by metallicity variations, providing a check for the $\mathrm{HCN} / \mathrm{HCO}^{+}$ ratio as a function of metallicity.

We have selected 3 positions in M 33 in/near the giant HII region NGC 604, clouds B8 B9 and B11 identified by Leroy et al. (2006) in IC 10, and Hubble V in NGC 6822 in which HCN was not detected (and $\mathrm{HCO}^{+}$not observed by Gratier et al. 2010a). Figures 1-3 show the positions and beamsizes of the observations on respectively IC 10, NGC 6822, and M 33 as seen at $24 \mu \mathrm{m}$ with Spitzer. For M 33, the positions observed in $\mathrm{HCN} / \mathrm{HCO}^{+}$by Buchbender et al. (2013) are also shown. We choose to show the $24 \mu \mathrm{m}$ emission because it is a high angular resolution tracer of star formation (e.g. Calzetti et al. 2010). Table 1 gives the sources, assumed distances, metallicities and positions of the new observations.

\section{Observations and data reduction}

\subsection{IRAM 30 m observations}

The IRAM 30 m telescope $^{1}$ was used in Nov/Dec 2014 and May 2015 to observe 3 positions in IC 10, the Hubble V HII region in NGC 6822, and a position in M 33 (M 33a). The coordinates are given in Table 1. The E090 and E230 dual polarization sidebandseparating EMIR receivers (Carter et al. 2012) were used to observe the lines listed in Table 2. The very broad FTS backends were used, providing $195 \mathrm{kHz}$ resolution (channel separation) over up to $16 \mathrm{GHz}$. The data were taken using the wobbler, with a throw of typically 2 arcmin, making sure not to risk having emission in the reference position. Telescope focus was checked at the beginning of each day and pointing was checked every 1.5-2 h. A line source, generally DR21 which is well-known to our group, was observed each day to check the tuning. The beamsize is about $28^{\prime \prime}$ at $90 \mathrm{GHz}, 22^{\prime \prime}$ for ${ }^{13} \mathrm{CO}(1-0)$ and $11^{\prime \prime}$ at the frequencies we have used in the $1 \mathrm{~mm}$ band.

System temperatures varied from $75-120 \mathrm{~K}$ for the lines near $90 \mathrm{GHz}$ to $100-160 \mathrm{~K}$ at $110 \mathrm{GHz}$ and $200 \mathrm{~K}$ at $115 \mathrm{GHz}$ and $\sim 300 \mathrm{~K}$ in the $1 \mathrm{~mm}$ band. Spectra were individually inspected and a small number of spectra identified as being of poor quality were dropped. The spectra for each line and source were then averaged and a continuum level (i.e. a zero-order baseline) subtracted if necessary. All spectra are presented on the main beam

\footnotetext{
1 Based on observations carried out with the IRAM $30 \mathrm{~m}$ Telescope. IRAM is supported by INSU/CNRS (France), MPG (Germany) and IGN (Spain).
} 


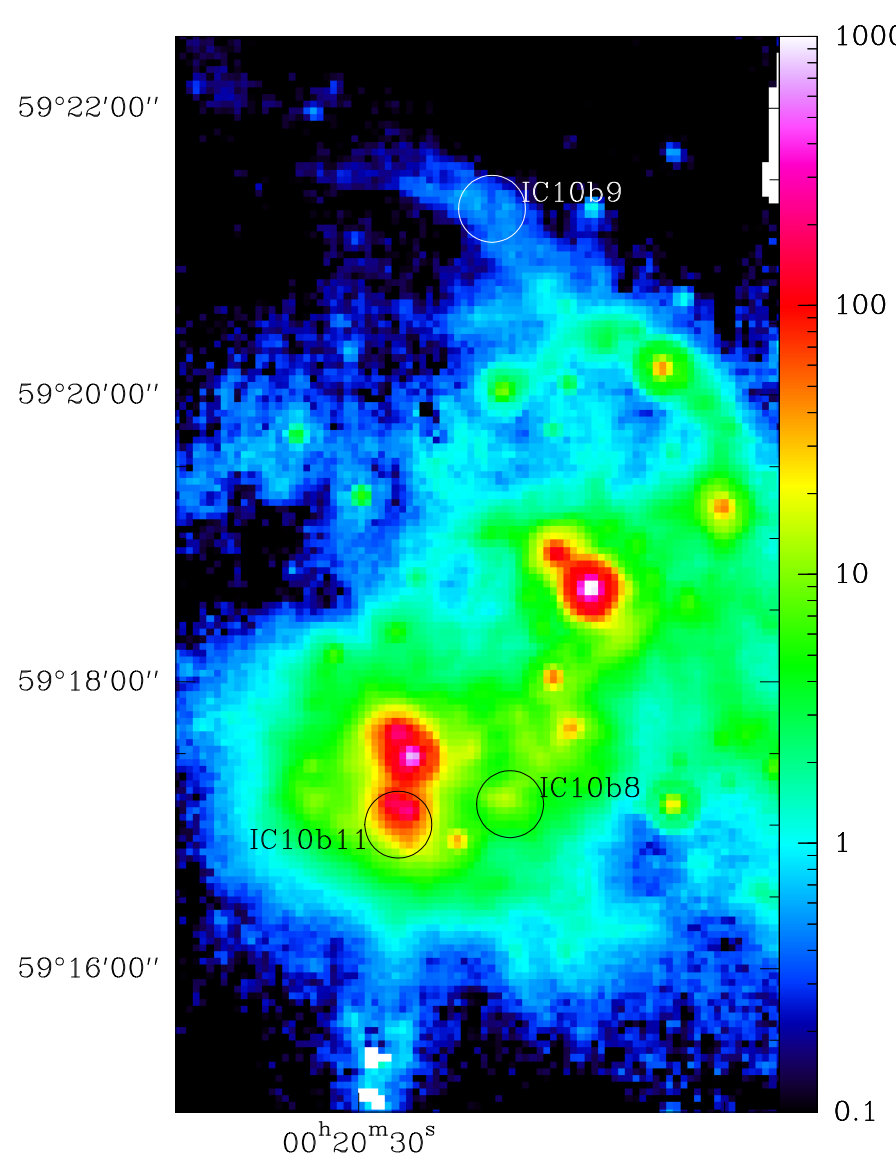

Fig. 1. Observed positions on a Spitzer $24 \mu \mathrm{m}$ map of IC 10. The circle size indicates the beamwidth of the observations. Units of the $24 \mu \mathrm{m}$ emission are $\mathrm{MJy} / \mathrm{sr}$ and the color scale is the same for Figs. 1-3 in order to facilitate comparison.

temperature scale. The forward efficiency of the telescope is 0.95 in the $3 \mathrm{~mm}$ band and 0.92 in the $1 \mathrm{~mm}$ band $(218-230 \mathrm{GHz})$. Conversion to $T_{\mathrm{mb}}$ was done using main beam efficiencies of 0.59 for ${ }^{12} \mathrm{CO}(2-1), 0.61$ for ${ }^{13} \mathrm{CO}(2-1)$ and $\mathrm{C}^{18} \mathrm{O}(2-1), 0.78$ for ${ }^{12} \mathrm{CO}(1-0)$ and ${ }^{13} \mathrm{CO}(1-0)$ and $\mathrm{C}^{18} \mathrm{O}(1-0), 0.80$ for $\mathrm{CS}(2-1)$, and 0.81 for $\mathrm{HCN}, \mathrm{HCO}^{+}, \mathrm{HNC}$, and $\mathrm{C}_{2} \mathrm{H}$. All data processing was done within the GILDAS environment ${ }^{2}$.

\subsection{Nobeyama radio observatory $45 \mathrm{~m}$ observations}

The Nobeyama $45 \mathrm{~m}$ observations took place from May 20th to June 1st, 2015 toward the GMCs in M 33 near NGC 604. The data were taken in the position-switching mode. The $\mathrm{TZ}$ receiver, which is a dual polarization sideband-separating SIS receiver, was used in combination with the Fast Fourier Transform Spectrometer SAM45. To cover the ${ }^{12} \mathrm{CO}(1-0),{ }^{13} \mathrm{CO}(1-0), \mathrm{C}^{18} \mathrm{O}(1-$ $0), \mathrm{HCO}^{+}(1-0), \mathrm{HCN}(1-0), \mathrm{C}_{2} \mathrm{H}(1-0), \mathrm{HNC}(1-0), \mathrm{SO}(2,3-1,2)$, $\mathrm{HC}_{3} \mathrm{~N}$ (10-9) frequencies, we used three frequency settings with a bandwidth of $8 \mathrm{GHz}(1 \mathrm{GHz} \times 8)$ and a frequency resolution of $244.14 \mathrm{kHz}$ in the dual polarization mode. Pointing was checked by observing the IRC+30021 $\mathrm{SiO}$ maser emission every hour, and was shown to be accurate within 3 arcsec. The main-beam efficiency was $38.8-50.0 \%$. The system noise temperature range was $140-500 \mathrm{~K}$. The beamsize of the NRO $45 \mathrm{~m}$ telescope is about $19^{\prime \prime}$ at $90 \mathrm{GHz}$ and $15^{\prime \prime}$ at $115 \mathrm{GHz}$.

\footnotetext{
2 http://www.iram.fr/IRAMFR/GILDAS/
}

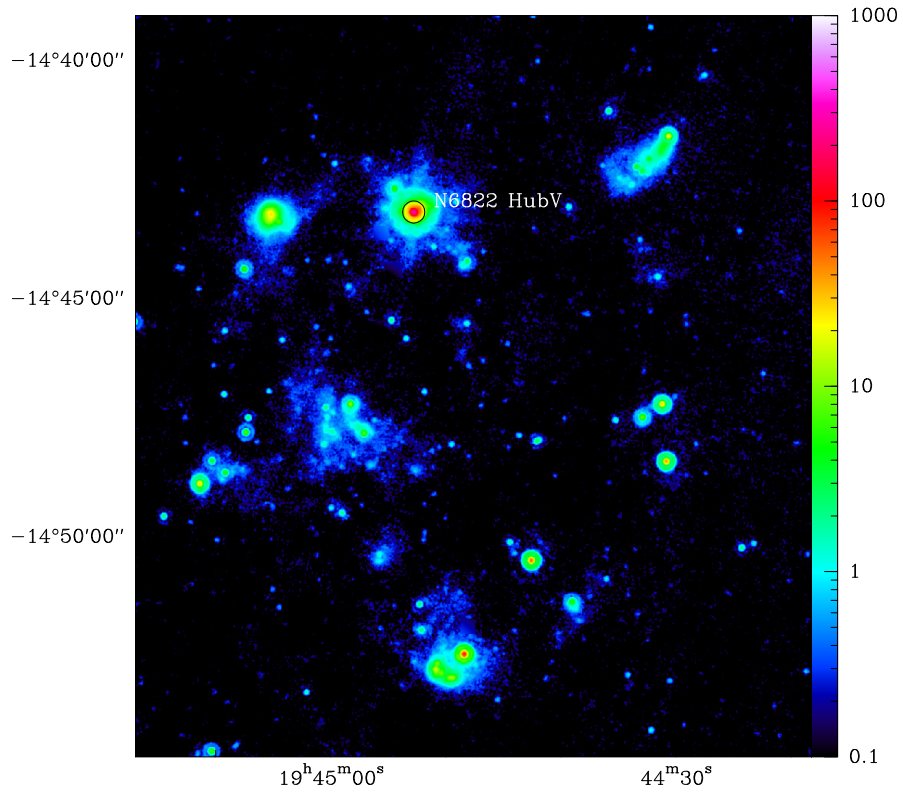

Fig. 2. Observed position on a Spitzer $24 \mu \mathrm{m}$ map of NGC 6822. The circle size indicates the beamwidth of the observations. Units of the $24 \mu \mathrm{m}$ emission are $\mathrm{MJy} / \mathrm{sr}$ and the color scale is the same for Figs. 1-3 in order to facilitate comparison.

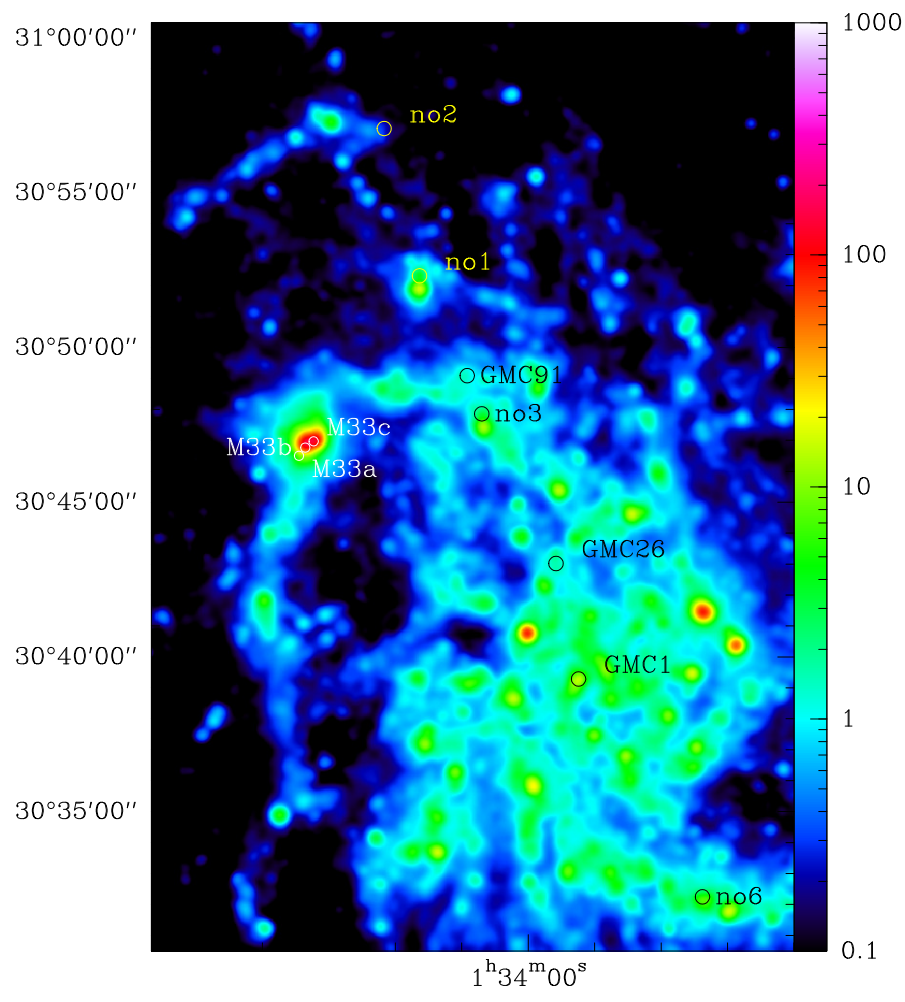

Fig. 3. Observed positions on a Spitzer $24 \mu \mathrm{m}$ map of M 33. The circle size indicates the beamwidth of the observations. Units of the $24 \mu \mathrm{m}$ emission are $\mathrm{MJy} / \mathrm{sr}$ and the color scale is the same for Figs. 1-3 in order to facilitate comparison. The positions and source names published in Buchbender et al. (2013) are shown as well.

We used the 4.3.1 version of the Common Astronomy Software Application (CASA) package (McMullin et al. 2007) to reduce the data obtained with the Nobeyama $45 \mathrm{~m}$. The CASA package is developed for the Atacama Large Millimeter/submillimeter Array (ALMA), and is also available for 
Table 2. HCN has two satellite (hyperfine) lines at relative velocities of -7.1 and $4.8 \mathrm{~km} \mathrm{~s}^{-1}$ and relative LTE intensities of 0.2 and 0.6 , respectively.

\begin{tabular}{ll}
\hline \hline Line & Frequency $(\mathrm{GHz})$ \\
\hline $\mathrm{HCN}(J=1-0)$ & 88.6318475 \\
$\mathrm{HCO}^{+}(J=1-0)$ & 89.18853 \\
$\mathrm{HNC}(J=1-0)$ & 90.66356 \\
$\mathrm{CS}(J=2-1)$ & 97.98095 \\
$\mathrm{~N}_{2} \mathrm{H}^{+}(J=1-0)$ & 93.1734 \\
$\mathrm{C}_{2} \mathrm{H}(N=1-0)$ & $87.316925^{a}$ \\
${ }^{13} \mathrm{CO}(J=1-0)$ & 110.20137 \\
$\mathrm{C}^{18} \mathrm{O}(J=1-0)$ & 109.78218 \\
${ }^{13} \mathrm{CO}(J=2-1)$ & 220.398686 \\
$\mathrm{C}^{18} \mathrm{O}(J=2-1)$ & 219.56036 \\
\hline
\end{tabular}

Notes. These lines are generally unresolved in all but the closest extragalactic sources. $\mathrm{HCO}^{+}, \mathrm{HNC}$, and $\mathrm{N}_{2} \mathrm{H}^{+}$have unresolved hyperfine structure. ${ }^{(a)} \mathrm{C}_{2} \mathrm{H}$ has a total of 6 lines 87.284156, 87.316925 (strongest), 87.328624 $(J=3 / 2 \rightarrow 1 / 2) 87.402004,87.407165$, $87.446512(J=1 / 2 \rightarrow 1 / 2)$.

reducing data obtained by a single-dish telescope such as the Nobeyama $45 \mathrm{~m}$ telescope. Here, we describe detailed steps of the data reduction process according to Shimajiri et al. (2015). In the first step, edge channels of each correlator band were flagged using the task sdflag2old, since the sensitivity of the edge channels drops. The SAM45 spectrometer of the Nobeyama $45 \mathrm{~m}$ telescope provides 4096 frequency channels, so that the data for the channel numbers from 1 to 300 and from 3796 to 4096 were flagged. In the second step, we determined the baseline of each spectrum using the task sdbaselineold. In the third step, we merged all the averaged spectra into one file using the task sdcoadd. In the final step, we applied the baseline correction again using the task sdbaselineold.

\section{Spectra and line ratios}

The line intensities observed as part of the new observations are presented in Table 3. In all cases, the $\mathrm{C}^{18} \mathrm{O}$ lines were not detected with noise levels that place the $\mathrm{C}^{18} \mathrm{O}$ at typically $10 \%$ or less of the ${ }^{13} \mathrm{CO}$ line intensity. The weakness of the $\mathrm{C}^{18} \mathrm{O}$ relative to the more abundant isotopes is even more extreme in the Magellanic Clouds (Chin et al. 1997, 1998), where ${ }^{13} \mathrm{CO} / \mathrm{C}^{18} \mathrm{O}$ line ratios are $\sim 40$, including some lower limits. In contrast, the line survey of two positions in M 51 carried out recently by Watanabe et al. (2014) showed ${ }^{13} \mathrm{CO} / \mathrm{C}^{18} \mathrm{O}$ line ratios of 4 although the ${ }^{12} \mathrm{CO} /{ }^{13} \mathrm{CO}$ line ratios are $\sim 10$, similar to those of the other galaxies. The weak $\mathrm{C}^{18} \mathrm{O}$ emission found in these galaxies, including $\mathrm{M} 33$, indicates that low ${ }^{18} \mathrm{O}$ abundance is a characteristic of subsolar metallicity environments although selective photodissociation of $\mathrm{C}^{18} \mathrm{O}$ likely further decreases the $\mathrm{C}^{18} \mathrm{O}$ emission (Shimajiri et al. 2014).

Figures 4-6 present the spectra of the main (detected) lines of interest $-{ }^{13} \mathrm{CO}(1-0), \mathrm{HCN}(1-0), \mathrm{HCO}^{+}(1-0), \mathrm{C}_{2} \mathrm{H}$, and ${ }^{12} \mathrm{CO}(1-0)$. It is immediately apparent that the $\mathrm{HCN}$ lines are weak relative to $\mathrm{HCO}^{+}$. This was already seen in the observations of the Magellanic Clouds presented by Chin et al. (1997, 1998). It was also noted for M 33 by Buchbender et al. (2013), as compared to M 31 (Brouillet et al. 2005) where the HCN and $\mathrm{HCO}^{+}$intensities are similar. Watanabe et al. (2014) find stronger $\mathrm{HCN}$ than $\mathrm{HCO}^{+}$emission near the nucleus of $\mathrm{M} 51$. Bigiel et al. (2016) found generally stronger $\mathrm{HCN}$ emission compared to $\mathrm{HCO}^{+}$in the inner disk of $\mathrm{M} 51$. The metallicity of M 51 is solar or somewhat higher and decreases with distance from the galactic center (Bresolin et al. 2004). Several clouds in the outskirts of M 51 were observed by Chen et al. (2016) who found similar $\mathrm{HCN}$ and $\mathrm{HCO}^{+}$fluxes (slightly stronger $\mathrm{HCO}^{+}$). All these works found that the HNC emission from M 51 was $20-50 \%$ of the $\mathrm{HCN}$ strength. This $\mathrm{HNC} / \mathrm{HCN}$ ratio is consistent with our data and those presented by Chin et al. (1997; and 1998) but higher than found by Buchbender et al. (2013) in their stacked spectrum.

The spectra presented by Watanabe et al. (2014) can be straightforwardly compared with our own. They detect the $\mathrm{N}_{2} \mathrm{H}^{+}$and HNCO lines at a level stronger than the $\mathrm{C}_{2} \mathrm{H}$ lines and $5-10 \%$ of the ${ }^{13} \mathrm{CO}(1-0)$ line strength. These lines are not detected in the low-metallicity systems. The $\mathrm{CN}$ lines are strong in $\mathrm{M} \mathrm{51,10-15 \%}$ of the ${ }^{13} \mathrm{CO}(1-0)$ line strength. The ${ }^{13} \mathrm{CO} /{ }^{12} \mathrm{CO}$ line ratio is nearly constant among the sources, almost irrespective of metallicity, so we choose these lines as a reference to compare relative line strengths. Neither we nor Buchbender et al. (2013) detect CN, even in stacked spectra. However, since we observed $\mathrm{CN}$ simultaneously with ${ }^{12} \mathrm{CO}(1-$ $0)$, a strong line, the limits are of order $10 \%$ of the ${ }^{13} \mathrm{CO}(1-0)$ intensity, and less constraining in some cases. The limits to the $\mathrm{CN}$ emission show, for the Buchbender et al. (2013) sources and IC 10 (the other upper limits in our data are not constraining), that it is weaker than $\mathrm{HCN}$ and $\mathrm{C}_{2} \mathrm{H}$ but consistent with the observations of the LMC by Chin et al. (1997).

In addition to $\mathrm{C}^{18} \mathrm{O}$, the weak and/or non-detected lines are all of nitrogen bearing molecules. Not only is the CO luminosity per $\mathrm{H}_{2}$ mass low in subsolar metallicity systems but the nitrogenbearing molecules are weak relative to $\mathrm{CO}$. The $\mathrm{C}_{2} \mathrm{H}$ lines on the other hand are not weak relative to $\mathrm{CO}$. Figure 7 shows the clearest of our $\mathrm{C}_{2} \mathrm{H}$ spectra, including most or all of the 6 components. While the signal-to-noise ratio is low for the weaker components, the fluxes are consistent with the ratios expected at local thermodynamic equilibrium (LTE).

Tercero et al. (2010) observed Orion KL with the IRAM $30 \mathrm{~m}$ telescope. As can be seen from their Fig. 2, the HCN and $\mathrm{H}^{13} \mathrm{CN}$ lines are much stronger than the $\mathrm{HCO}^{+}$and $\mathrm{H}^{13} \mathrm{CO}^{+}$ lines respectively. These lines are just an example as generally, in this gas exposed an extremely high radiation field, the line ratios are different both from M 51 and lower metallicity sources. Nishimura et al. (2016) observed position IC 10b11 with the NRO $45 \mathrm{~m}$ and obtain similar detections and non-detections.

\section{Source of the variations in line ratios}

$\mathrm{HCN}$ and $\mathrm{HCO}^{+}$have similar dipole moments and are both linear molecules, with a substitution of $\mathrm{O}^{+}$for $\mathrm{N}$ in the latter. It is thus reasonable to expect a link between the N/O abundance ratio and the abundance ratio of the two molecules.

For the range in metallicities of our sources, the N/O abundance ratio is roughly proportional to the oxygen abundance $(\mathrm{O} / \mathrm{H})$ although the N/O ratio appears roughly constant for lower metallicities (see Figs. 3-4 and 5 in Pilyugin et al. 2003; van Zee \& Haynes 2006, respectively). This is attributed to a change in the origin of nitrogen from primary at low metallicity to secondary at higher metallicity.

Figure 8 shows the variation of the $\mathrm{HCN} / \mathrm{HCO}^{+}$line intensity ratio. Both of these lines are optically thick in dense regions of the Galaxy but at these metallicities and scales (10-100 pc), the lines are expected to be optically thin, with the possible exception of M 31. In Fig. 8, each galaxy has been assumed to have the same metallicity for all positions. M 33 and M 31 only have weak 
Table 3. New observations presented in this work.

\begin{tabular}{|c|c|c|c|c|c|c|c|c|}
\hline Source & $I_{\mathrm{CO}}$ & $I_{13} \mathrm{CO}$ & $I_{\mathrm{C}^{18} \mathrm{O}}$ & $I_{\mathrm{HCO}^{+}}$ & $I_{\mathrm{HCN}}$ & $I_{\mathrm{HNC}}$ & $I_{\mathrm{CCH}}$ & $I_{\mathrm{CS}}$ \\
\hline M 33a ${ }^{a}$ & & $0.64 \pm .07$ & $0.04 \pm .065$ & $0.20 \pm .05$ & $0.12 \pm .05$ & $\lesssim 0.06$ & $0.09 \pm .05$ & \\
\hline M 33a ${ }^{b}$ & $15.7 \pm .6$ & $0.58 \pm .10$ & $0.21 \pm .12$ & $0.212 \pm .037$ & $0.083 \pm .039$ & $0.031 \pm .036$ & $0.117 \pm .048$ & \\
\hline M $33 b^{b}$ & $15.5 \pm .5$ & $1.16 \pm .12$ & $\lesssim 0.11$ & $0.806 \pm .055$ & $0.339 \pm .048$ & $0.208 \pm .046$ & $0.434 \pm .068$ & \\
\hline $\mathrm{M} 33 \mathrm{c}^{b}$ & $12.1 \pm .4$ & $0.73 \pm .08$ & $\lesssim 0.09$ & $0.659 \pm .046$ & $0.496 \pm .046$ & $\lesssim 0.051$ & $0.075 \pm .060$ & \\
\hline $\mathrm{IC} 10 \mathrm{~b} 8^{a}$ & $5.54 \pm .08$ & $0.55 \pm .023$ & $\lesssim 0.014$ & $0.157 \pm .009$ & $0.032 \pm .010$ & $0.025 \pm .009$ & $0.031 \pm .009$ & $\lesssim 0.04$ \\
\hline IC $10 \mathrm{~b} 9^{a}$ & $5.93 \pm .08$ & $0.46 \pm .03$ & $\lesssim 0.02$ & $0.04 \pm .02$ & $\lesssim 0.015$ & $\lesssim 0.016$ & $\lesssim 0.014$ & $0.06 \pm .03$ \\
\hline $\mathrm{IC} 10 \mathrm{~b} 11^{a}$ & $14.9 \pm .1$ & $1.12 \pm .04$ & $0.11 \pm .036$ & $0.718 \pm .023$ & $0.245 \pm .025$ & $0.088 \pm .027$ & $0.21 \pm .03$ & $0.26 \pm .05$ \\
\hline $\mathrm{N}^{6} 822 \mathrm{HubV}^{a}$ & $2.0 \pm 0.12^{c}$ & $0.16 \pm .02$ & $\lessgtr 0.019$ & $0.115 \pm .012$ & $0.025 \pm .011$ & $\lesssim 0.013$ & $0.049 \pm .012$ & \\
\hline
\end{tabular}

Notes. All fluxes are on the main beam temperature scale, ${ }^{(a)}$ indicates observatons with the IRAM $30 \mathrm{~m}$ antenna and ${ }^{(b)}$ with the NRO $45 \mathrm{~m}$ telescope; ${ }^{(c)}$ taken from Gratier et al. (2010a). The other lines within the bandpasses such as $\mathrm{SO}, \mathrm{HC}_{3} \mathrm{~N}$, or $\mathrm{N}_{2} \mathrm{H}^{+}$, were not detected. $\mathrm{C}^{18} \mathrm{O}$ was not detected either but was included in this table to directly compare with ${ }^{13} \mathrm{CO}$. Blank parts of the table indicate that the line was not observed. Limits are given as §the $1 \sigma$ noise level to distinguish between a tentative line (e.g. HCN in N6822HubV, $0.25 \pm .011 \mathrm{~K} \mathrm{~km} \mathrm{~s}^{-1}$ ) and something where there is no sign of a line (typically intensity below $1 \sigma$ or negative).

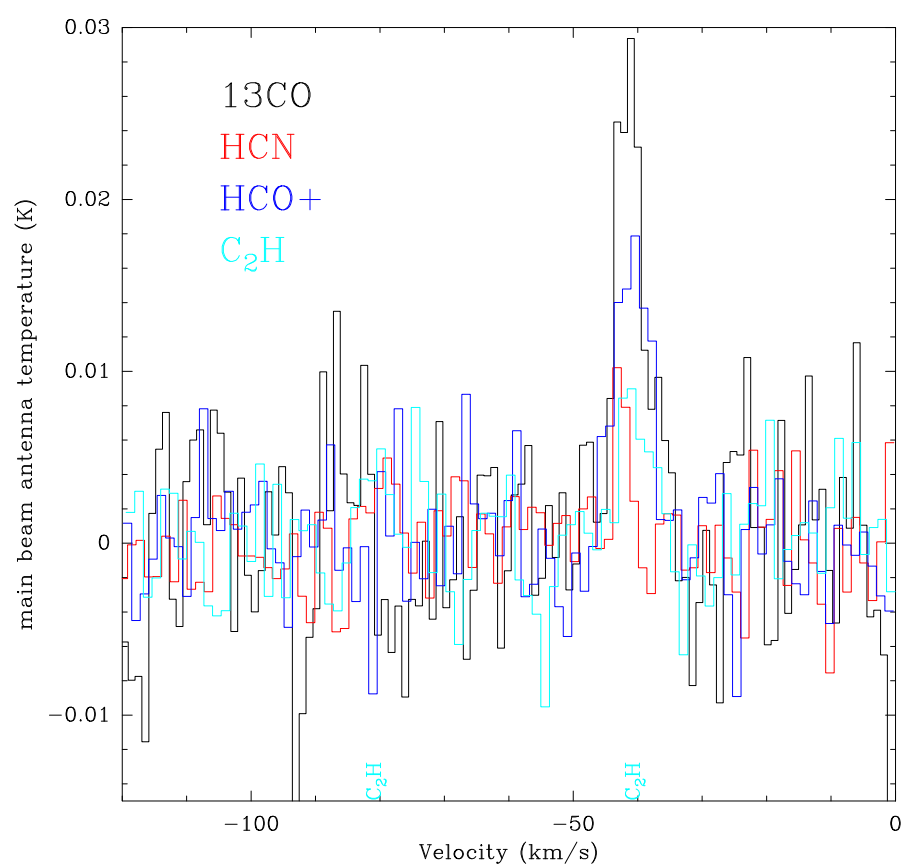

Fig. 4. ${ }^{13} \mathrm{CO}$ and dense gas tracers as observed in the Hubble V HII region in NGC 6822. Color coding is indicated in the panel.

metallicity gradients (Magrini et al. 2009; Sanders et al. 2012) and the others have no known gradient although there may be metallicity inhomogeneities. An increase in the $\mathrm{HCN} / \mathrm{HCO}^{+}$line intensity ratio from roughly $1 / 4$ at a metallicity of 0.3 solar to about 0.9 in M 31 follows this trend in N/O abundance. At these scales, we have no reason to invoke other sources than the N/O abundance ratio to explain the observed trend in the $\mathrm{HCN} / \mathrm{HCO}^{+}$ line intensity ratio with metallicity. The N/O abundance ratio was also proposed by Anderson et al. (2014) as a possible explanation of the low $\mathrm{HCN} / \mathrm{HCO}^{+}$ratio in the $\mathrm{LMC}$.

\section{Link with star formation}

Figure 9 shows the link between the dense gas fraction, as traced by the intensity ratios of $\mathrm{HCN}$ and $\mathrm{HCO}^{+}$to $\mathrm{CO}$, and the SFR as traced by the $24 \mu \mathrm{m}$ emission. When the $24 \mu \mathrm{m}$ emission is strong, both the $\mathrm{HCN} / \mathrm{CO}$ and $\mathrm{HCO}^{+} / \mathrm{CO}$ ratios are high and the relation appears monotonic. However, the $\mathrm{HCO}^{+} / \mathrm{CO}$ ratio shows less scatter between the galaxies (M 33 and IC 10) than the $\mathrm{HCN} / \mathrm{CO}$ line ratio, presumably due to the lesser effect of metallicity variations which cause the $\mathrm{HCN} / \mathrm{CO}$ ratios of IC 10 to be lower than in M 33. Therefore, at the $100 \mathrm{pc}$ scale, it appears that $\mathrm{HCO}^{+}$may be the preferred tracer of dense gas in low-metallicity objects.

Buchbender (2014) observed $\mathrm{HCN}, \mathrm{HCO}^{+}$, and $\mathrm{CO}$ in cloud no3 in M 33 at a resolution of about 20 pc. From his Fig. 11.3, clear differences at this scale are seen between the distribution of the two lines. His Fig. 11.5 shows a comparison of the HCN and $\mathrm{HCO}^{+}$maps with various star formation tracers.

The observations presented here are at a scale of $100 \mathrm{pc}$ (range of 70-140 pc), intermediate between the subpc-scale Galactic data and the typically kpc or whole-galaxy extragalactic measurements. Figure 10 shows how the $\mathrm{HCN}$ and $\mathrm{HCO}^{+}$luminosities compare with Galactic and extragalactic observations at various scales. Points above the line can be considered to have weak line emission and those below to have strong line emission. The trend is basically a linear relation between the emission of the dense gas tracers and IR luminosity, presumably reflecting a proportionality between the mass of dense gas and the SFR. However, and this is why we have chosen to separate galactic centers and inner disks from outer regions, the physical conditions in large galaxies are such that stellar surface densities and molecular fractions are systematically higher in the inner disk as compared to external regions (Chen et al. 2015; Usero et al. 2015). Furthermore, spirals show radial metallicity gradients.

Looking at the data points above and below the line showing HCN-IR proportionality, it is immediately clear that $\mathrm{HCN}$ is relatively stronger in the inner regions (Chen et al. 2015; Krips et al. 2008) and weaker in low-metallicity galaxies (points from Chin et al. 1997, 1998; Buchbender et al. 2013, and this work). Turning to the $\mathrm{HCO}^{+}$emission in the lower panel, the linear relation appears to have less systematic scatter in that the low- $Z$ points are not off the global fit and inner disk points are closer to it. However, fewer extragalactic $\mathrm{HCO}^{+}$data are available. Figure 11 expands the vertical scale by plotting the ratio of the IR-to-HCN (or $\mathrm{HCO}^{+}$) luminosity as a function of the IR luminosity. Here it becomes apparent that at the small scales observed by Ma et al. (2013) and Wu et al. (2005), the IR emission increases more rapidly than the $\mathrm{HCN}$ or $\mathrm{HCO}^{+}$. This could be due to either an increase in the line optical depth, causing saturation for the more massive clumps, or, as suggested by Wu et al. (2010), that the IR luminosity of low-mass star forming regions decreases more quickly due to an incomplete sampling of the IMF such that few (if any) massive stars are present. 


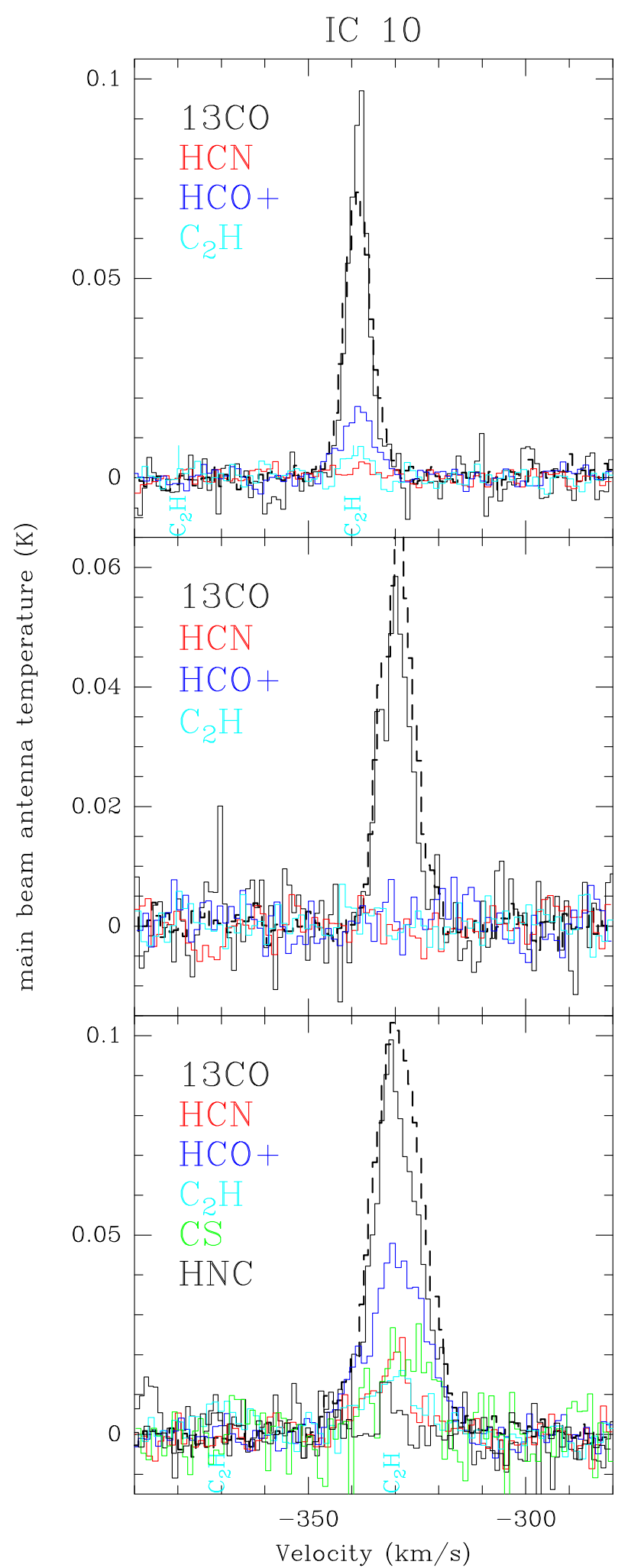

Fig. 5. ${ }^{13} \mathrm{CO},{ }^{12} \mathrm{CO}$ and dense gas tracers as observed in the b8, b9, and b11 regions in IC 10. Color coding is indicated in the panel. Note that additional lines were detected in IC $10 \mathrm{~b} 11$. The ${ }^{12} \mathrm{CO}$ line scale (dashed) is divided by 10 .

One would expect high redshift objects to have generally subsolar metallicities and, due to their necessarily young ages, low N/O abundance ratios (much of the nitrogen production occurs after a substantial time delay). The observations of highredshift objects, necessarily very bright, by Gao et al. (2007)

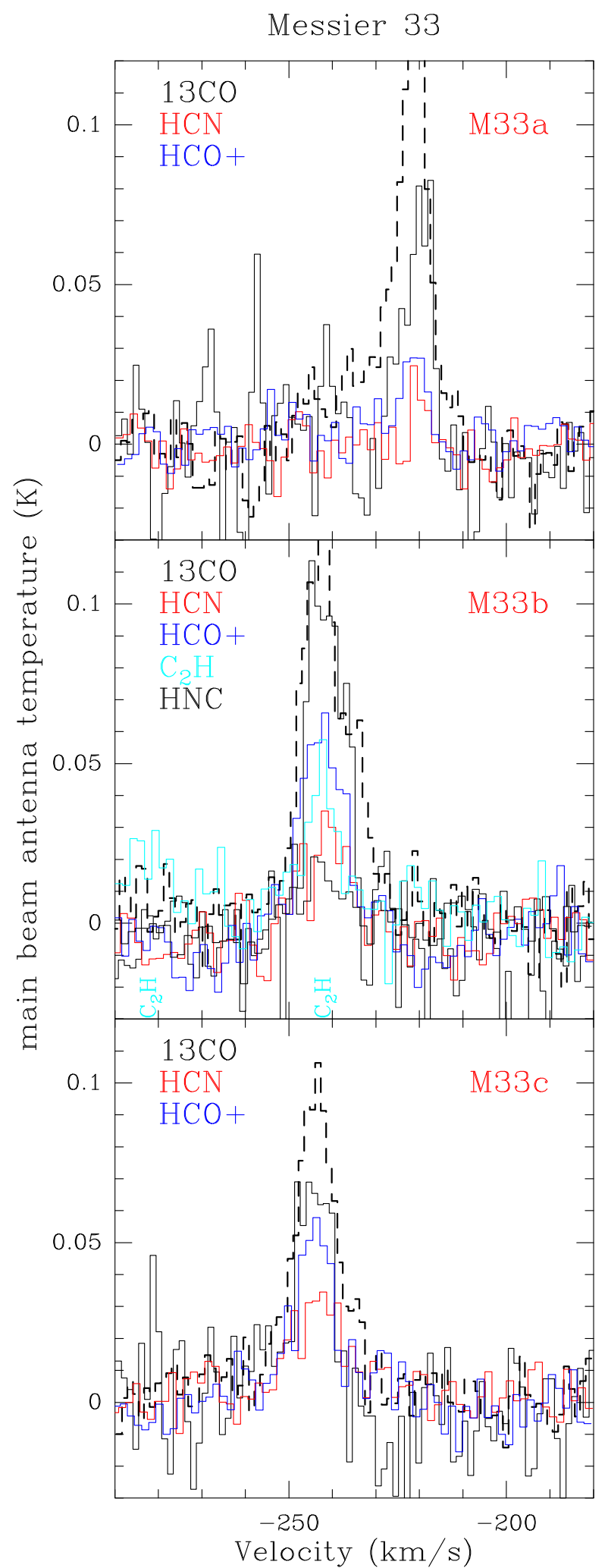

Fig. 6. ${ }^{13} \mathrm{CO},{ }^{12} \mathrm{CO}$ and dense gas tracers as observed in the $\mathrm{M} 33 \mathrm{a}$, $M 33 b$, and $M$ 33c regions in $M$ 33, all near the giant HII region NGC 604. Color coding is indicated in the panel. Note that additional lines were detected in $\mathrm{M} 33 \mathrm{~b}$. The ${ }^{12} \mathrm{CO}$ line scale (dashed) is divided by 10 .

seem to go in this direction as the HCN emission per unit IR luminosity is weak (or equivalently the IR emission is strong). The HCN/IR ratio is about a factor of 2 weaker compared to that established in local galaxies, yet this value is similar to 


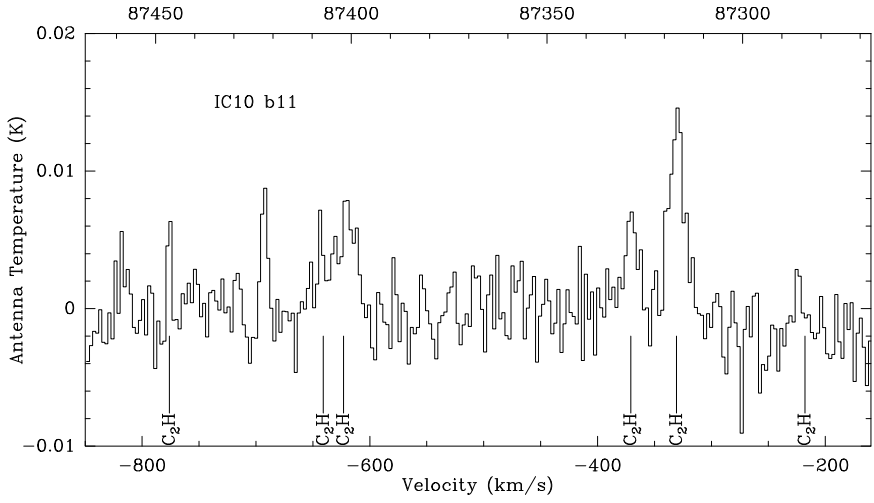

Fig. 7. $\mathrm{C}_{2} \mathrm{H}$ spectrum observed in source b11 in the galaxy IC 10. Upper scale gives the rest frequency in MHz. The 6 transitions are marked. The flux ratios are compatible with LTE.

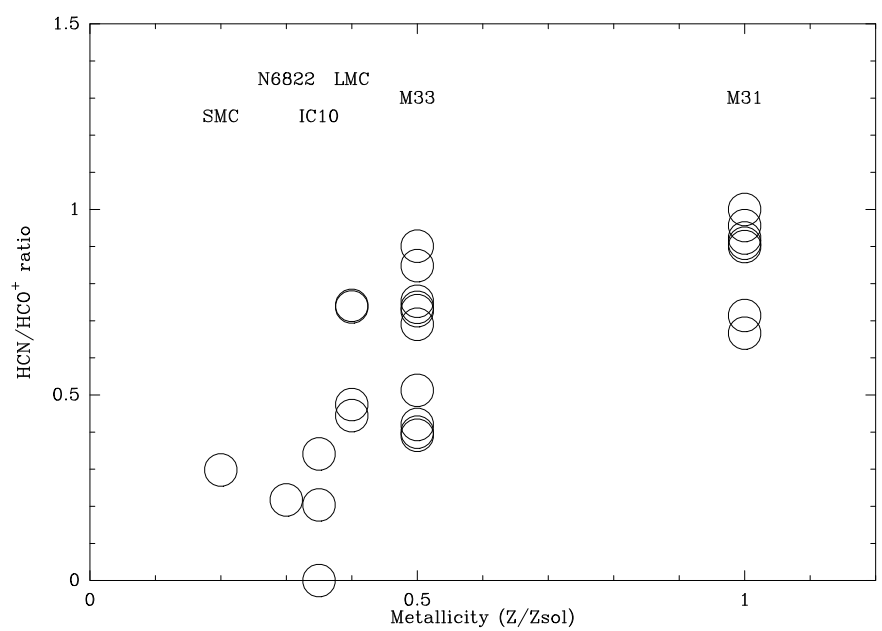

Fig. 8. Variation of the $\mathrm{HCN} / \mathrm{HCO}^{+}$ratio with metallicity. References are Brouillet et al. (2005), Chin et al. (1997, 1998) for M 31 and the Magellanic Clouds, Buchbender et al. (2013) and the present work for M 33, and this work for IC 10 and NGC 6822. Typical uncertainties for individual points are 0.2 dex for the metallicity and 0.3 in the $\mathrm{HCN} / \mathrm{HCO}^{+}$ratio.

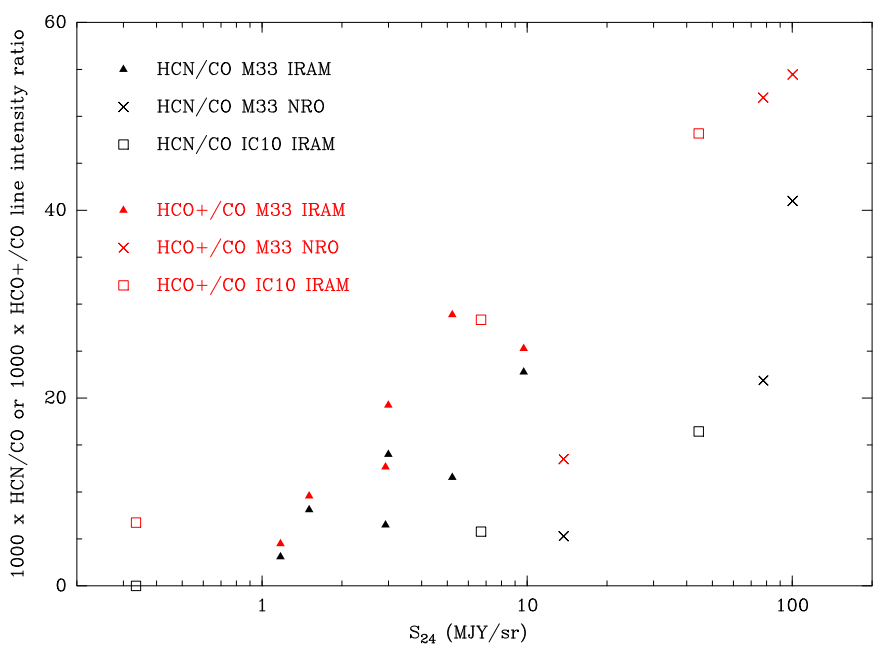

Fig. 9. Link between dense gas fraction and SFR. The HCN/CO and $\mathrm{HCO}^{+} / \mathrm{CO}$ ratios are used to trace the fraction of dense molecular gas and the $24 \mu \mathrm{m}$ intensity is used as a proxy for the SFR. $\mathrm{HCN} / \mathrm{CO}$ is in black and $\mathrm{HCO}^{+} / \mathrm{CO}$ is in red. Observations are from Buchbender et al. (2013) and the present work. Typical uncertainties are $20 \%$ for $\mathrm{HCO}^{+} / \mathrm{CO}$ and $25 \%$ for the $\mathrm{HCN} / \mathrm{CO}$ ratio.
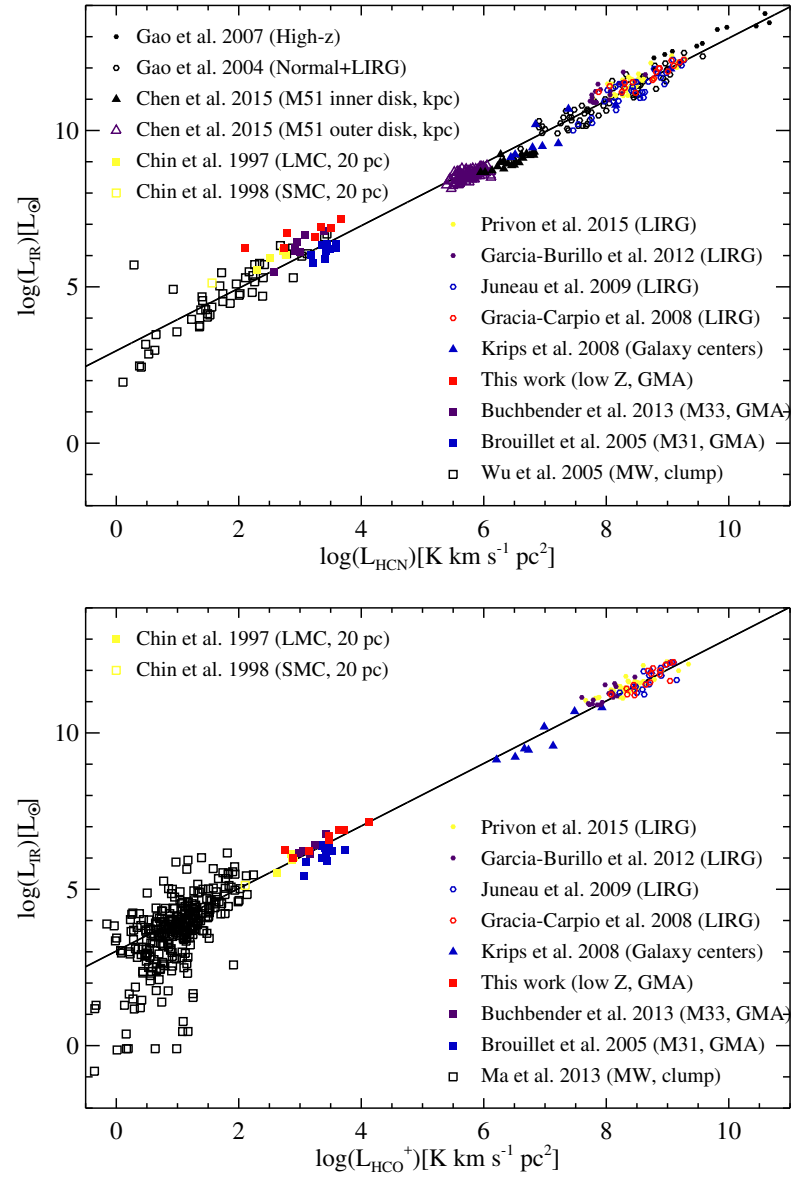

Fig. 10. Link between IR luminosity and HCN (upper panel) and $\mathrm{HCO}^{+}$(lower panel) luminosity. The lines show a linear relation between the two quantities. The fits $\operatorname{are} \log \left(L_{\mathrm{IR}}\right)=2.95424+\log \left(L_{\mathrm{HCN}}\right)$ and $\log \left(L_{\mathrm{IR}}\right)=3.04258+\log \left(L_{\mathrm{HCO}^{+}}\right)$and are from respectively Gao \& Solomon (2004b) and Graciá-Carpio et al. (2008). The symbols indicate the reference and the scale of the observation.

what we found here in nearby low- $Z$ galaxy systems. Nevertheless, the $\mathrm{HCN} / \mathrm{CO}$ ratios of the low- $Z$ systems and highredshift AGNs/ULIRGs are at the two extreme ends, the smallest $\mathrm{HCN} / \mathrm{CO}$ values are found in low- $Z$ whereas the highest are in high redshift AGNs/ULIRGs, similar to that of local ULIRGs despite their higher HCN/IR ratio. It is still unclear whether low N/O abundance ratios could explain all the differences. Therefore, the low $\mathrm{HCN} / \mathrm{IR}$ ratio at high redshift might suggest a higher SFR per unit dense molecular gas in high-redshift AGNs/ULIRGs, although not necessarily so in nearby low- $Z$ systems, compared to those of local normal star-forming galaxies. $\mathrm{HCO}^{+}$measurements would greatly help to determine the appropriate interpretation.

\section{Summary and conclusions}

The low-metallicity Local Group galaxies NGC 6822, IC 10, and M 33 have been observed in a number of molecular lines, including the dense gas tracers $\mathrm{HCN}(1-0)$ and $\mathrm{HCO}^{+}(1-0)$, at a scale of $70-140 \mathrm{pc}$. In addition to ${ }^{12,13} \mathrm{CO}, \mathrm{HCO}^{+}, \mathrm{HCN}, \mathrm{HNC}, \mathrm{C}_{2} \mathrm{H}$, and $\mathrm{CS}(2-1)$ have been detected in one or more objects.

The $\mathrm{HCN}$ and HNC lines are weak with respect to the IR or ${ }^{12,13} \mathrm{CO}$ emission but the $\mathrm{HCO}^{+}$follows the trends observed in solar metallicity galaxies. $\mathrm{C}^{18} \mathrm{O}, \mathrm{N}_{2} \mathrm{H}^{+}, \mathrm{CN}$, and $\mathrm{HNCO}$ were not detected although in M 51 (Watanabe et al. 2014) these lines are stronger than $\mathrm{C}_{2} \mathrm{H}$, which was well detected. N/O varies as 

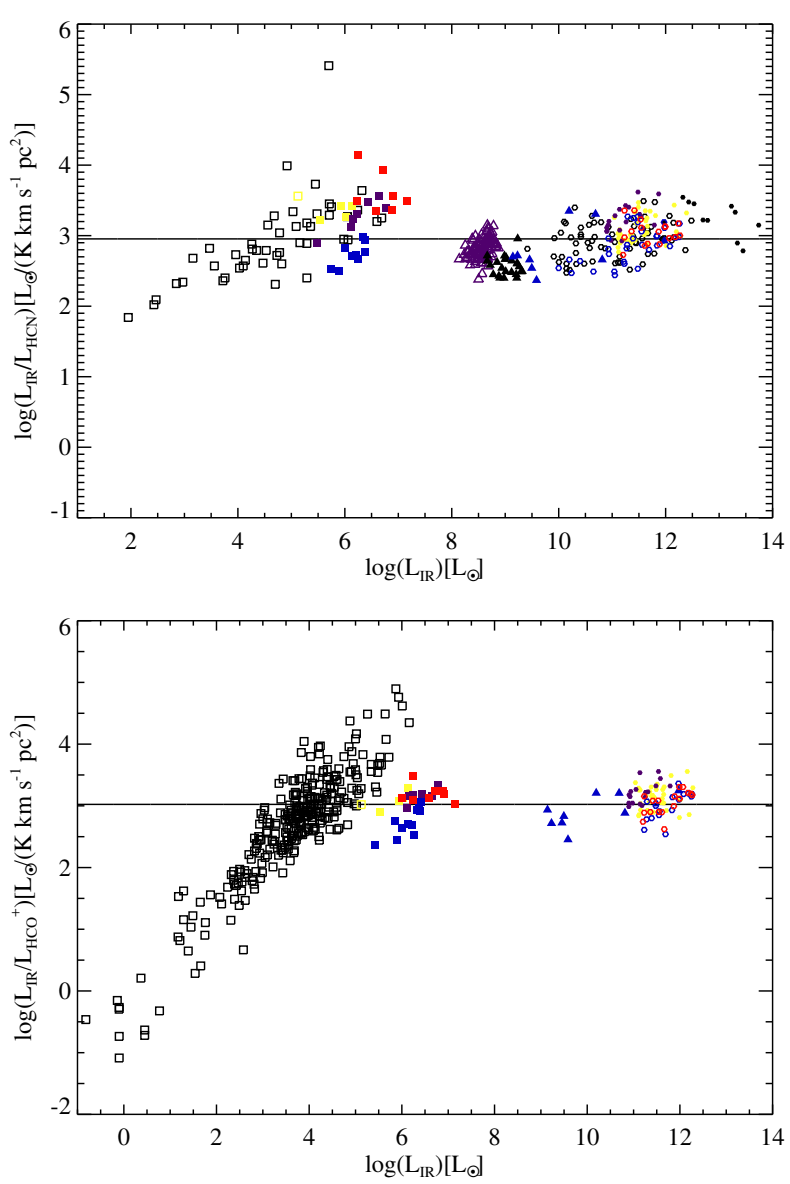

Fig. 11. Ratio between IR and $\mathrm{HCN}$ (upper panel) and $\mathrm{HCO}^{+}$(Lower panel) luminosities as a function of IR luminosity. The goal here is to see finer structure than in the previous figure as the vertical scale has been greatly expanded. References are as given in Fig. 10.

$\mathrm{O} / \mathrm{H}$ at the metallicities explored here. An accordingly low nitrogen abundance could explain the weakness of the nitrogenbearing species in our observations and more generally in the observations of low metallicity galaxies.

The SFR, as traced by the $24 \mu \mathrm{m}$ emission, increases with the dense gas fraction, as traced by the $\mathrm{HCN} / \mathrm{CO}$ or $\mathrm{HCO}^{+} / \mathrm{CO}$ line ratios.

The low- $Z$ points fall below (i.e. less $\mathrm{HCN}$ ) the linear $\mathrm{HCN}-\mathrm{IR}$ Galactic-extragalactic luminosity correlation but right on the $\mathrm{HCO}^{+}-\mathrm{IR}$ correlation. When measuring the dense gas mass of low metallicity objects, $\mathrm{HCO}^{+}$may be a more robust tracer than $\mathrm{HCN}$.

Acknowledgements. J.B. would like to thank Laura Magrini for very useful discussions about abundances and abundance gradients in galaxies. We would also like to thank Belen Tercero for the data enabling us to calculate the line ratios of interest in Orion KL. The authors would like to acknowledge support from the French Agence Nationale de Recherche (ANR) grant ANR-11-BS56-010 for the STARFICH project (www.obs.u-bordeaux1.fr/ webformationetoiles/StarFichE) and the European Research Council under the European Union's Seventh Framework Programme (ERC Advanced Grant Agreement No. 291294 - "ORISTARS"). Y.G. acknowledges support from the National Natural Science Foundation of China (NSFC grants 11390373 and 11420101002), the Strategic Priority Research Program "The Emergence of Cosmological Structures" (grant XDB09000000) and Key Research Program of Frontier Sciences of the Chinese Academy of Sciences.

\section{References}

Anderson, C. N., Meier, D. S., Ott, J., et al. 2014, ApJ, 793, 37

André, P., Men'shchikov, A., Bontemps, S., et al. 2010, A\&A, 518, L102

André, P., Di Francesco, J., Ward-Thompson, D., et al. 2014, Protostars and Planets VI, 27

Baan, W. A., Henkel, C., Loenen, A. F., Baudry, A., \& Wiklind, T. 2008, A\&A, 477, 747

Bigiel, F., Leroy, A. K., Jiménez-Donaire, M. J., et al. 2016, ApJ, 822, L26

Boquien, M., Calzetti, D., Combes, F., et al. 2011, AJ, 142, 111

Bresolin, F., Garnett, D. R., \& Kennicutt, Jr., R. C. 2004, ApJ, 615, 228

Brouillet, N., Muller, S., Herpin, F., Braine, J., \& Jacq, T. 2005, A\&A, 429, 153

Buchbender, C. 2014, Ph.D. Thesis, Universidad de Granada, Spain, http:// hdl.handle.net/10481/31707

Buchbender, C., Kramer, C., Gonzalez-Garcia, M., et al. 2013, A\&A, 549, A17

Calzetti, D., Wu, S.-Y., Hong, S., et al. 2010, ApJ, 714, 1256

Carter, M., Lazareff, B., Maier, D., et al. 2012, A\&A, 538, A89

Chen, H., Gao, Y., Braine, J., \& Gu, Q. 2015, ApJ, 810, 140

Chen, H., Braine, J., Gao, Y., Koda, J., \& Gu, Q. 2016, ApJ, submitted

Chin, Y.-N., Henkel, C., Whiteoak, J. B., et al. 1997, A\&A, 317, 548

Chin, Y.-N., Henkel, C., Millar, T. J., Whiteoak, J. B., \& Marx-Zimmer, M. 1998, A\&A, 330, 901

Druard, C., Braine, J., Schuster, K. F., et al. 2014, A\&A, 567, A118

Galametz, M., Kennicutt, R. C., Calzetti, D., et al. 2013, MNRAS, 431, 1956

Galleti, S., Bellazzini, M., \& Ferraro, F. R. 2004, A\&A, 423, 925

Gao, Y., \& Solomon, P. M. 2004a, ApJS, 152, 63

Gao, Y., \& Solomon, P. M. 2004b, ApJ, 606, 271

Gao, Y., Carilli, C. L., Solomon, P. M., \& Vanden Bout, P. A. 2007, ApJ, 660, L93

García-Burillo, S., Usero, A., Alonso-Herrero, A., et al. 2012, A\&A, 539, A8

Graciá-Carpio, J., García-Burillo, S., Planesas, P., Fuente, A., \& Usero, A. 2008, A\&A, 479, 703

Gratier, P., Braine, J., Rodriguez-Fernandez, N. J., et al. 2010a, A\&A, 512, 68

Gratier, P., Braine, J., Rodriguez-Fernandez, N. J., et al. 2010b, A\&A, 522, 3

Heiderman, A., Evans, II, N. J., Allen, L. E., Huard, T., \& Heyer, M. 2010, ApJ, 723, 1019

Hirota, T., Yamamoto, S., Mikami, H., \& Ohishi, M. 1998, ApJ, 503, 717

Hunter, D. A. 2001, ApJ, 559, 225

Juneau, S., Narayanan, D. T., Moustakas, J., et al. 2009, ApJ, 707, 1217

Kepley, A. A., Leroy, A. K., Frayer, D., et al. 2014, ApJ, 780, L13

Krips, M., Neri, R., García-Burillo, S., et al. 2008, ApJ, 677, 262

Kuno, N., Nakai, N., Handa, T., \& Sofue, Y. 1995, PASJ, 47, 745

Lada, C. J., Lombardi, M., \& Alves, J. F. 2010, ApJ, 724, 687

Lada, C. J., Forbrich, J., Lombardi, M., \& Alves, J. F. 2012, ApJ, 745, 190

Leroy, A., Bolatto, A., Walter, F., \& Blitz, L. 2006, ApJ, 643, 825

Liu, F., \& Gao, Y. 2010, ApJ, 713, 524

López-Sepulcre, A., Cesaroni, R., \& Walmsley, C. M. 2010, A\&A, 517, A66

Ma, B., Tan, J. C., \& Barnes, P. J. 2013, ApJ, 779, 79

Magrini, L., \& Gonçalves, D. R. 2009, MNRAS, 398, 280

Magrini, L., Stanghellini, L., \& Villaver, E. 2009, ApJ, 696, 729

Mateo, M. L. 1998, ARA\&A, 36, 435

McMullin, J. P., Waters, B., Schiebel, D., Young, W., \& Golap, K. 2007, in Astronomical Data Analysis Software and Systems XVI, eds. R. A. Shaw, F. Hill, \& D. J. Bell, ASP Conf. Ser., 376, 127

Meier, D. S., \& Turner, J. L. 2005, ApJ, 618, 259

Nishimura, Y., Shimonishi, T., Watanabe, Y., et al. 2016, ApJ, 829, 94

Pilyugin, L. S., Thuan, T. X., \& Vílchez, J. M. 2003, A\&A, 397, 487

Privon, G. C., Herrero-Illana, R., Evans, A. S., et al. 2015, ApJ, 814, 39

Sanders, N. E., Caldwell, N., McDowell, J., \& Harding, P. 2012, ApJ, 758, 133

Shimajiri, Y., Kitamura, Y., Saito, M., et al. 2014, A\&A, 564, A68

Shimajiri, Y., Sakai, T., Kitamura, Y., et al. 2015, ApJS, 221, 31

Skillman, E. D., Kennicutt, R. C., \& Hodge, P. W. 1989, ApJ, 347, 875

Tennekes, P. P., Harju, J., Juvela, M., \& Tóth, L. V. 2006, A\&A, 456, 1037

Tercero, B., Cernicharo, J., Pardo, J. R., \& Goicoechea, J. R. 2010, A\&A, 517, A96

Usero, A., Leroy, A. K., Walter, F., et al. 2015, AJ, 150, 115

van Zee, L., \& Haynes, M. P. 2006, ApJ, 636, 214

Watanabe, Y., Sakai, N., Sorai, K., \& Yamamoto, S. 2014, ApJ, 788, 4

Wu, J., Evans, II, N. J., Gao, Y., et al. 2005, ApJ, 635, L173

Wu, J., Evans, II, N. J., Shirley, Y. L., \& Knez, C. 2010, ApJS, 188, 313 